\title{
Morphological Transformations for Enhancement of Images with Poor Contrast and Detection of Background
}

\author{
Manisha G Tekam \\ ABHA COE, Butibori, Nagpur
}

\begin{abstract}
This paper deals with enhancement of images with poor contrast and detection of background. Proposes a frame work which is used to detect the background in images characterized by poor contrast. Image enhancement has been carried out by the two methods based on the Weber's law notion. The first method employs information from image background analysis by blocks, while the second transformation method utilizes the opening operation, closing operation, which is employed to define the multi-background gray scale images. The complete image processing is done using MATLAB simulation model. Finally, this paper is organized as follows as Morphological transformation and Weber's law. Image background approximation to the background by means of block analysis in conjunction with transformations that enhance images with poor lighting. The multi background notion is introduced by means of the opening by reconstruction shows a comparison among several techniques to improve contrast in images. Histogram for every method is shown. Finally, conclusions are presented.
\end{abstract}

Keyword: Image Background Analysis by blocks, Morphological Methods, Weber's law notion, Opening Operation, Closing Operation, Erosion-Dilation method, Histogram equalization, Block Analysis for Gray level images.

\section{INTRODUCTION}

The image enhancement problem in digital images can be approached from various methodologies, among which is mathematical morphology (MM). Such operators consist in accordance to some proximity criterion, in selecting for each point of the analyzed image, a new grey level between two patterns (primitives) [1], [4]. Even though morphological contrast has been largely studied, there are no methodologies, from the point of view MM, capable of simultaneously normalizing and enhancing the contrast in images with poor lighting. On the other side, one of the most common techniques in image processing to enhance dark regions is the use of nonlinear functions, such as logarithm or power functions ; otherwise, a method that works in the frequency domain is the homomorphism filter. In addition, there are techniques based on data statistical analysis, such as global and local histogram equalization. However, the main disadvantage of histogram equalization is that the global properties of the image cannot be properly applied in alocal context, frequently producing a poor performance in detail preservation. This paper deals with the detection of background in images with poor contrast. The complete image processing is done using MATLAB simulation model. Also, some operators to enhance and normalize the contrast in grey level images with poor lighting are introduced. Contrast operators are based on the logarithm function in a similar way to Weber's law the use of the logarithm function avoids abrupt changes in lighting. Also, two approximations to compute the background in the processed images are proposed. The first proposal consists in an analysis by blocks, whereas in the second proposal, the opening by reconstruction is used. A comparison among several techniques to improve contrast in images is shown. Histogram for each result is shown.

Finally, conclusions are presented.

\section{MORPHOLOGICAL TRANSFORMATIONS AND WEBER'S LAW}

\subsection{Morphology}

Morphology is a technique of image processing based on shape and form of objects. Morphological methods apply a structuring element to an input image, creating an output image at the same size. The value of each pixel in the input image is based on a comparison of the corresponding pixel in the input image with its neighbors. The morphological operations can first be defined on grayscale images where the source image is planar (single-channel). The definition can then be expanded to full-colour images.

\subsection{Morphological Operations}

Morphological operations such as erosion, dilation, opening, and closing. Often combinations of these operations are used to perform morphological image analysis [3], [17]. Irrespective of the size of the structuring element, the origin is located at its centre. Morphological opening $\gamma_{\mu \mathrm{B}}(f)(x)$ is and Morphologicalclosing is $\varphi_{\mu \mathrm{B}}(f)(x)$

$$
\begin{aligned}
\gamma_{\mu \mathrm{B}}(f)(x) & =\delta_{\mu \mathrm{B}}\left(\varepsilon_{\mu \mathrm{B}}(f)\right)(x) \\
\varphi_{\mu \mathrm{B}}(f)(x) & =\varepsilon_{\mu \mathrm{B}}\left(\delta_{\mu \mathrm{B}}(f)\right)(x)
\end{aligned}
$$

Where $\square \square$ a homothetic parameter, size is $\square \square$ means a square of ( $2 \square \square \square 1) \square(2 \square \square \square 1)$ pixels. B is the structuring element of size $3 \times 3$ (here $\square \square=1$ ).

\section{Dilation}

Dilation is a transformation that produces an image that is the same shape as the original, but is a different size. 
Dilation stretches or shrinks the original figure. Dilation Closing operation

increases the valleys and enlarges the width of maximum Closing of an image is the reverse of opening operation. regions, so it can remove negative impulsive noises but do little on positives ones. The dilation of $\mathrm{A}$ by the structuring element $\mathrm{B}$ is defined by:

$$
A \oplus B=\bigcup_{b \in B} A_{b}
$$

The structuring element $\mathrm{s}$ is positioned with its origin at $(\mathrm{x}, \mathrm{y})$ and the new pixel value is determined using the rule:

$$
g(x, y)= \begin{cases}1 & \text { if } s \text { hits } f \\ 0 & \text { otherwise }\end{cases}
$$

The following figure illustrates the morphological dilation of a gray scale image. The dilation function applies the appropriate rule to the pixels in the neighborhood and assigns a value to the corresponding pixel in the output image. In the figure, the morphological dilation function sets the value of the output pixel to 16 because it is the maximum value of all the pixels in the input pixel's neighborhood defined by the structuring element is on.

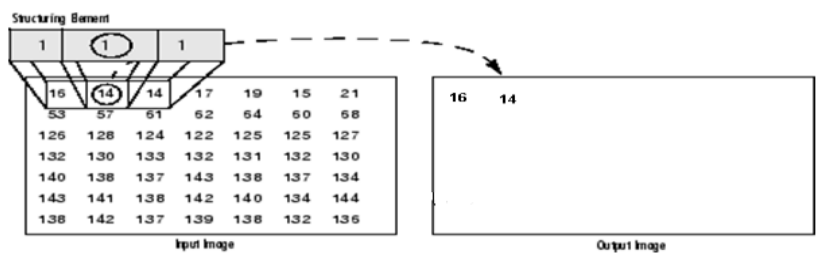

Figure 1.Morphological Dilation of a Gray scale Image

\section{Erosion}

It is used to reduce objects in the image and known that erosion reduces the peaks and enlarges the widths of minimum regions, so it can remove positive noises but affect negative impulsive noises little. The erosion of the binary image $\mathrm{A}$ by the structuring element $\mathrm{B}$ is defined by:

$$
\mathrm{A} ! \mathrm{B}=\{\{\mathrm{z} \in \mathrm{E} \mid \mathrm{B} \approx \subseteq \mathrm{A}\}
$$

Erosion of image $\mathrm{f}$ by structuring element $\mathrm{s}$ is given by

$$
\mathrm{f} \ominus \mathrm{S}
$$

The structuring element $\mathrm{s}$ is positioned with its origin at $(\mathrm{x}, \mathrm{y})$ and the new pixel value is determined using the rule:

$$
g(x, y)=\left\{\begin{array}{cc}
1 & \text { if } s \text { hits } f \\
0 & \text { otherwise }
\end{array}\right.
$$

The following figure illustrates the morphological erosion of a gray scale image.

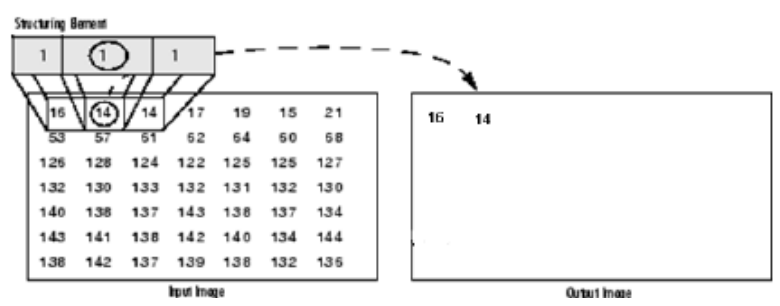

Figure4. Morphological Erosion of a Grayscale Image

\section{Opening operation}

The opening of A by B is obtained by the erosion of A by $\mathrm{B}$, followed by dilation of the resulting image by $\mathrm{B}$ :

$$
A \circ B=(A ! B) \oplus B
$$

In the case of the square of side 10 , and a disc of radius 2 as the structuring element, the opening is a square of side 10 with rounded corners, where the corner radius is 2 . The sharp edges start to disappear.
The closing of A by B is obtained by the dilation of A by $\mathrm{B}$, followed by erosion of the resulting structure by $\mathrm{B}$ :

$$
A \cdot B=(A \oplus B) ! B
$$

\section{Existing System}

The range of intensity i.e. the difference between highest and lowest intensity values in an image gives a measure of its contrast. There are standard techniques like histogram equalization, histogram stretching for improving the poor contrast of the degraded image [11]. However there is a need for devising context-sensitive techniques based on local contrast variation since the image characteristics differ considerably from one region to another in the same image and also the local histogram does not necessarily follow the global histogram. The enhancement level is not significant and provides good results only for certain images but fails to provide good results for most of the images, especially those taken under poor lighting. In other words, it doesn't provide good performance for detail preservation.

\section{Proposed System}

In a method to enhance contrast is proposed; the methodology consists in solving an optimization problem that maximizes the average local contrast of an image. The optimization formulation includes a perceptual constraint derived directly from human threshold contrast sensitivity function. Here, the basic idea is to select a set of training images which look good perceptually, next a Gaussian mixture model for the color distribution in the face region is built, and for any given input image, a color tone mapping is performed so that the color statistics in the face region matches the training examples. In this way, even though the reported algorithms to compensate changes in lighting are varied, some are more adequate than others.

\section{Weber's Law}

The study of contrast sensitivity has dominated visual perception research. In psycho-visual studies, the contrast $\mathrm{C}$ of an object with luminance $\mathrm{L} \max$ against its surrounding luminance L min is defined as follows

$$
C=\frac{\operatorname{Lmax}-\operatorname{Lmin}}{\operatorname{Lmin}}
$$

C - Contrast of the image, Lmax - Luminance of the image ad Lmin - Luminance of the surroundings If $\mathrm{L}=\mathrm{Lmin}$ and $\Delta=\mathrm{Lmax}-\mathrm{Lmin}$ so it can be written as follows

$$
C=\frac{\Delta L}{L}
$$

On the other hand, in, a methodology to compute the background parameter was proposed. In this paper, an approximation to Weber's law [14] is considered by taking the luminance $\mathrm{L}$ as the grey level intensity of a function (image); Equation (9) indicates that $\Delta(\log \mathrm{L})$ is proportional to C; therefore Weber's law can be expressed

$$
C=k \log L+b \quad L>0
$$

This law has a logarithmic relation. This technique is applied to image processing to enhance the image effectively. Where ' $C$ ' is the contrast, ' $k$ ' and ' $b$ ' are constants, ' $b$ ' being the background parameter and ' $k$ ' 
being the scaling factor for enhancement.In our case, an approximation to Weber's law is considered by taking the luminance $\mathrm{L}$ as the grey level intensity of a function (image); in this way, expression (10) is written as

$$
C=k \log f+b f>0
$$

\section{Block Analysis:}

\section{For Gray level images:}

Let $\mathrm{f}$ be the original image which is subdivided into number of blocks with each block is the sub-image of the original image. For each analyzed block, maximum $\left(\mathrm{M}_{\mathrm{i}}\right)$ and minimum $\left(\mathrm{m}_{\mathrm{i}}\right)$ values are used to determine the background criteria $\tau_{\mathrm{i}}$ in the following way: $\tau_{i}=\frac{m_{i}+M_{i}}{2} \quad \forall i=1,2, \ldots, n_{m}$

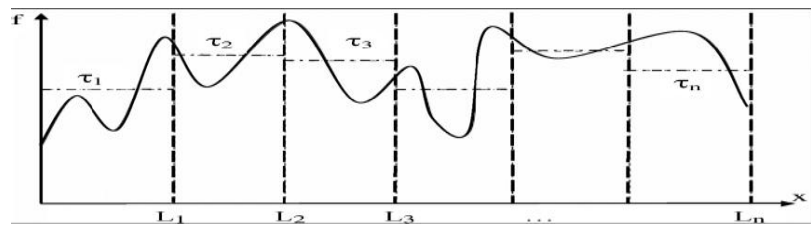

Fig. 2.1.2: Background criteria obtained by block analysis

Once $\tau_{\mathrm{i}}$ is calculated, this value is used to select the background parameter associated with the analyzed block. As follows, an expression to enhance the contrast is proposed:

$$
\Gamma_{\tau_{i}}(f)= \begin{cases}k_{i} \log (f+1)+M_{i}, & f \leq \tau_{i} \\ k_{i} \log (f+1)+m_{i}, & \text { otherwise. }\end{cases}
$$

Note that the background parameter depends on the $\tau_{\mathrm{i}}$ value. If $\mathrm{f}<=\tau_{\mathrm{i}}$ (dark region), the background parameter takes the value of the maximum intensity $\left(\mathrm{M}_{\mathrm{i}}\right)$ within the analyzed block, and the minimum intensity $\left(\mathrm{m}_{\mathrm{i}}\right)$ value otherwise. On the other hand, since grey level images are used in this work, the constant $\mathrm{K}_{\mathrm{i}}$ in above equation is obtained as follows:

With

$$
\begin{aligned}
& m_{i}^{*}= \begin{cases}m_{i}, & f>\tau_{i} \\
M_{i}, & f \leq \tau_{i} .\end{cases} \\
& k_{i}=\frac{255-m_{i}^{*}}{\log (256)} \quad \forall i=1,2, \ldots, n .
\end{aligned}
$$

On the other hand, $\mathrm{M}_{\mathrm{i}}$ and $\mathrm{m}_{\mathrm{i}}$ values are used as background parameters to improve the contrast depending on the $\tau_{\mathrm{i}}$ value, due to the background is different for clear and dark regions. The more is the number of blocks; the better will be quality of the enhanced image. In the enhanced images, it can be seen that the objects that are not clearly visible in the original image are revealed. The results are best obtained by keeping the size of the structuring element as $2(\mu=2)$. Sample input (left half of the image) and output image (right half) for block analysis is shown below

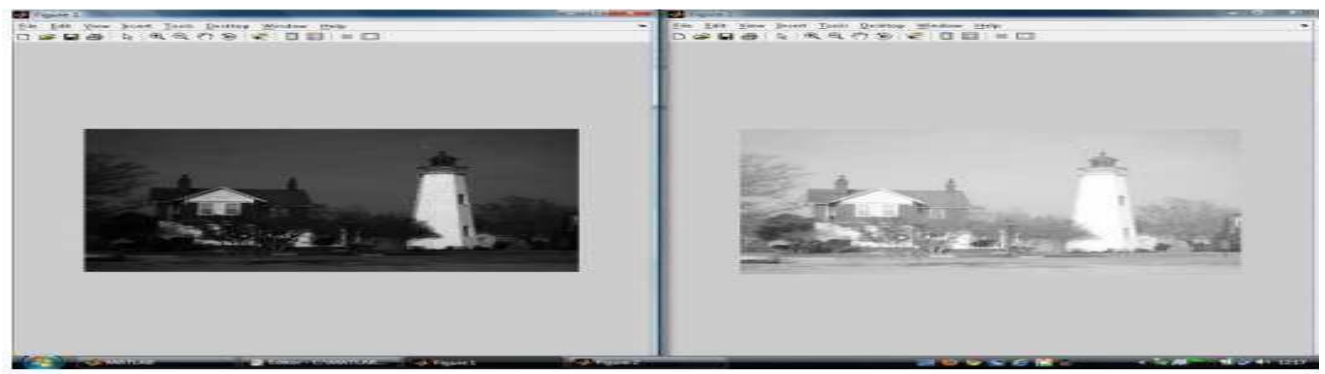

Figure7. Block Analysis $(\mu=2)$

Erosion:

Erosion is one of the two basic operators in the area of mathematical morphology, the other being dilation. The erosion operator takes two pieces of data as inputs. The first is the image which is to be eroded. The second is a (usually small) set of coordinate points known as a structuring element (also known as a kernel). In erosion, every object pixel that is touching a background pixel is changed into a background pixel.

\section{Dilation:}

Dilation adds pixels to the boundaries of objects in an image. In dilation, every background pixel that is touching an object pixel is changed into an object pixel. Let $\mathrm{I}_{\max }(\mathrm{x})$ and $\mathrm{I}_{\min }(\mathrm{x})$ be the maximum and minimum intensity values taken from one set of pixels contained in a window (B) of elemental size (3 X 3 elements), $\mathrm{x}$ belongs to $\mathrm{D}$. Notice that the window corresponds to the structuring element .A new expression can be derived as shown:

$$
\tau(x)=\frac{I_{\min }(x)+I_{\max }(x)}{2}
$$

Where $I_{\max }(x)$ and $I_{\min }(x)$ values correspond to the morphological dilation and erosion defined by the orderstatistical filters. Thus, the above expression is expressed

$$
\tau(x)=\frac{\varepsilon_{\mu}(f)(x)+\delta_{\mu}(f)(x)}{2} .
$$

Finally the proposed transformation is expressed as

$$
\begin{array}{r}
\Gamma_{\tau(x)}(f)=\left\{\begin{array}{rr}
k_{\tau(x)} \log (f+1)+\delta_{\mu}(f)(x), & f \leq \tau(x) \\
k_{\tau(x)} \log (f+1)+\varepsilon_{\mu}(f)(x), & \text { otherwise }
\end{array}\right. \\
\text { and } k_{\tau(x)}=\frac{255-\tau(x)}{\log (256)} .
\end{array}
$$

By employing Erosion-Dilation method we obtain a better local analysis of the image for detecting the background criteria than the previously used method of Blocks.

The trouble with this method is that morphological erosion or dilation when used with large size of $\square$ to reveal the background, undesired values maybe generated.

Image Background Analysis Using Opening by Reconstruction for gray scale images

This method is similar to block analysis in many ways; 
apart from the fact that the manipulation is done on the This particular characteristic allows the modification of image as a whole rather than partitioning it into blocks. the altitude of regional maxima when the size of the Firstly minimum $I_{\min }(\mathrm{x})$ and maximum intensity $I_{\max }(\mathrm{x})$ structuring element increases thereby aiding in detection contained in a structuring element (B) of elemental size 3 of the background criteria as follows:

$\times 3$ is calculated.

$$
\tau(x)=\gamma_{\mu B}(f)(x)
$$

The above obtained values are used to find the background Where opening by reconstruction is expressed as criteria $\tau_{i}$ as described below

$$
\tau_{(x)}=\frac{I_{\min }(x)+I_{\max }(x)}{2}
$$

$$
\gamma_{\mu B}(f)(x)=\lim _{n \rightarrow \infty} \delta_{f}^{n}\left(\varepsilon_{\mu B}(f)\right)(x)
$$

It can be observed from the above equation that opening Where Imin (x) and Imax (x) corresponds to morphological erosion and dilation respectively, Therefore

$$
\tau(x)=\frac{\varepsilon_{\mu}(f)(x)+\delta_{\mu}(f)(x)}{2}
$$

In this way the contrast operator can be described as in equations (12) and (13). By employing Erosion-Dilation method we obtain a better local analysis of the image for detecting the background criteria than the previously used method of Blocks. By increasing the size of the structuring element more pixels will be taken into account for finding the background criteria [9].

In general it is desirable to filter an image without generating any new components. We go for opening by reconstruction because it restores the original shape of the objects in the image that remain after erosion as it touches the regional minima and merges the regional maxima by reconstruction first erodes the input image and uses it as a marker. Background parameter $\mathrm{b}(\mathrm{x})$ is calculated by eroding the above obtained background criterion $\tau(\mathrm{x})$ which is described below

$$
b(x)=\varepsilon_{1}\left[\gamma_{\mu}(f)\right](x)
$$

Contrast enhancement is obtained by applying Weber's law as expressed below

$$
\begin{gathered}
\xi_{\gamma \mu}(\mathrm{f})=\mathrm{k}(\mathrm{x}) \log (\mathrm{f}+1)+\varepsilon_{1}\left[\gamma_{\mu}(\mathrm{f})\right] \\
\mathrm{k}(\mathrm{x})=\frac{\operatorname{maxint}-\varepsilon_{1}\left[\gamma_{\mu}(\mathrm{f})\right]}{\log (\max \operatorname{int}+1)}
\end{gathered}
$$

Where, max int refers to maximum gray level intensity which is equal to 255 . If the intensity of the background increases, the image becomes lighter because of the additive effect of the whiteness (i.e. maximum intensity) of the background.

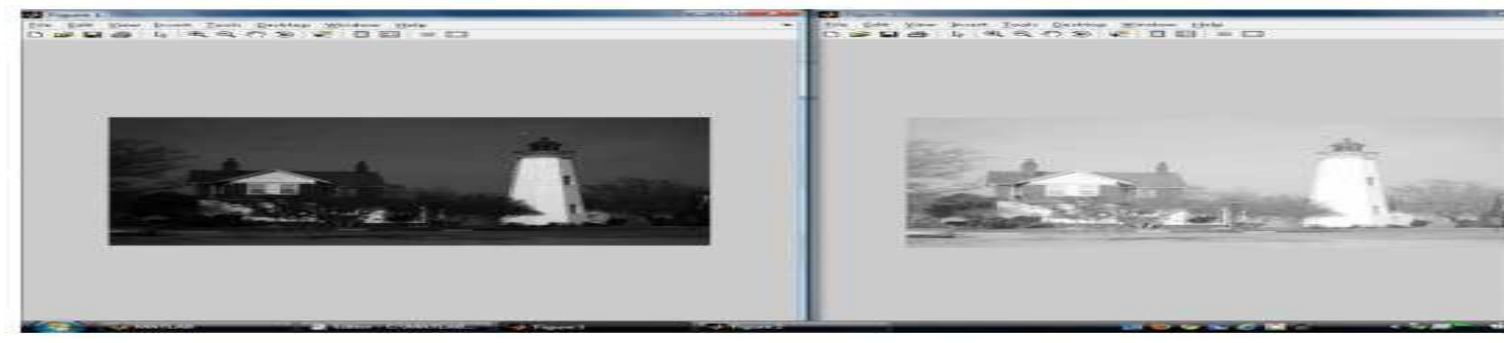

Figure8. Closing By Reconstruction $(\mu=2)$

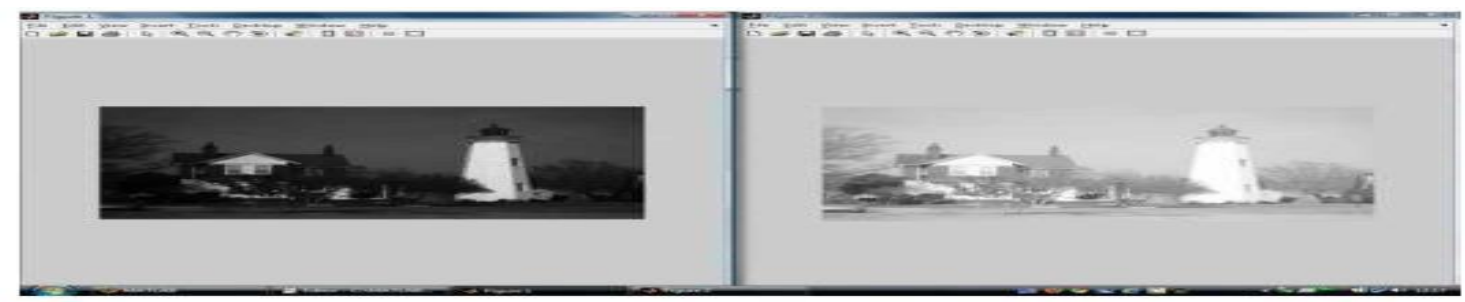

Figure10. Opening by Reconstruction $(\mu=2)$

3. SIMULATION RESULTS
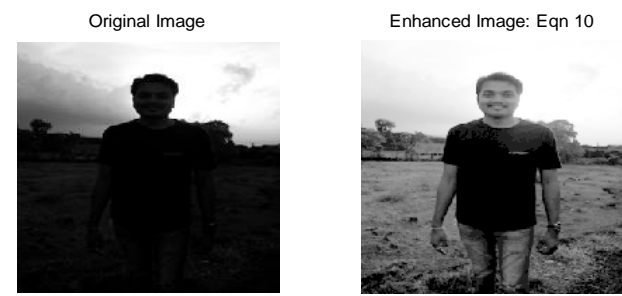

Fig (a).original image Fig (b).enhanced image
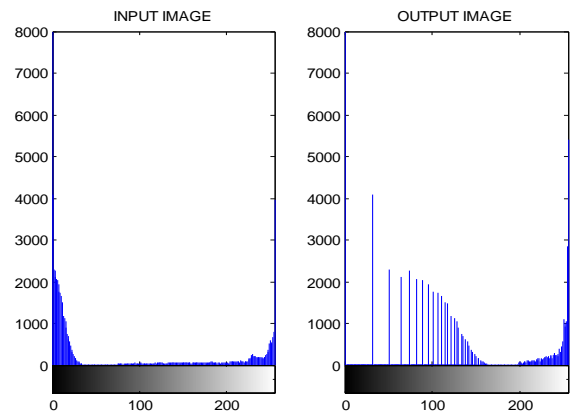

Fig(c) Histogram by analysis of block 

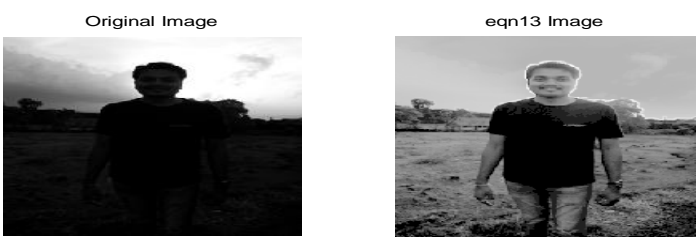

Fig (a).original image fig (b).erosion and dilation
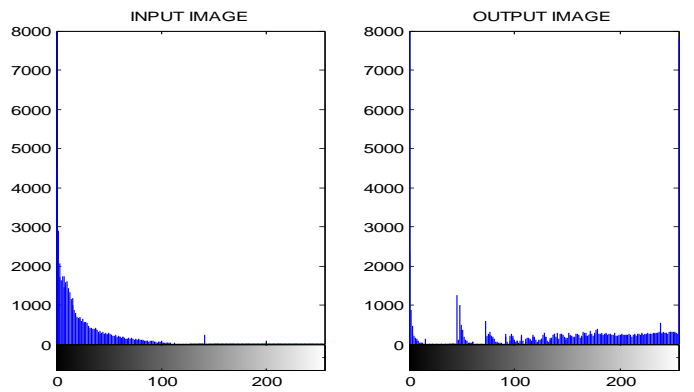

Fig (c) Histogram
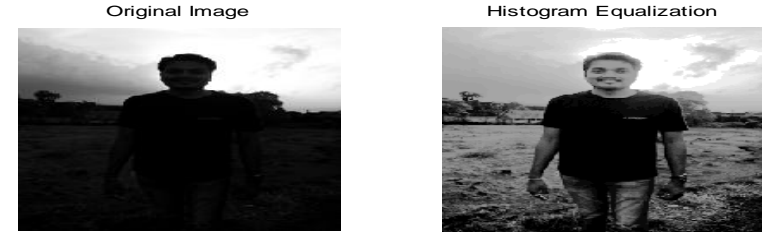

Fig (a).original image Fig (b). histogram equalization
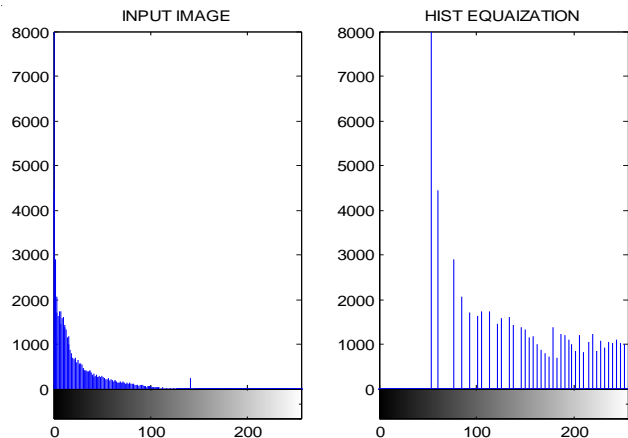

Fig(c) Histogram
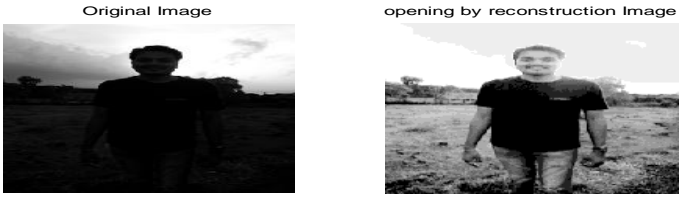

Fig(a). original image

Fig (b). opening by reconstruction
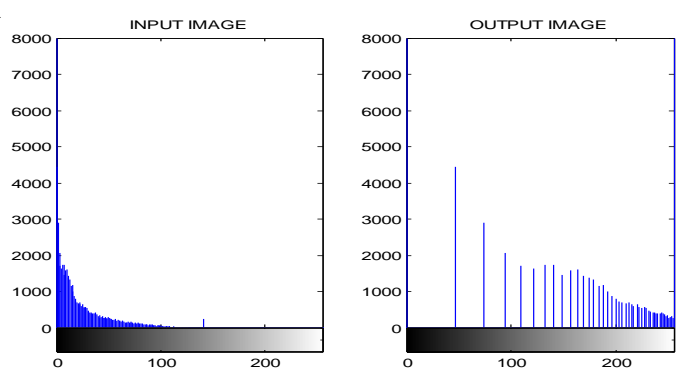

fig(c) Histogram

\section{CONCLUSION}

First, a methodology was introduced to compute an approximation to the background using blocks analysis. This proposal was subsequently extended using mathematical morphology operators. However, a difficulty was detected when the morphological erosion and dilation were employed; therefore, a new proposal to detect the image background was propounded, that is based on the use of morphological connected transformations. Also, morphological contrast enhancement transformations were introduced. Such operators are based on Weber's law notion. The performances of the proposals provided in this work were illustrated by means of several examples throughout the paper. Also, the operators performance employed in this paper were compared with others given in the literature. Finally, a disadvantage of contrast enhancement transformations studied in this paper is that they can only be used satisfactorily in images with poor lighting; in a future work this problem will be considered.

\section{REFERENCES}

[1]. I. R. Terol-Villalobos, "A multiscale contrast approach on Morphological connected contrast mappings" Opt. Eng., vol. 43, no. 7, pp. 1577-1595, 2009.

[2]. J. Kasperek, "Real time morphological image contrast enhancement in FPGA," in LNCS, New York: Springer, 2008

[3]. I.R. Terol-Villalobos, "Morphological image enhancement and segmentation with analysis," P. W. Hawkes, Ed. New York: Academic, 2005, pp. 207-273.

[4]. F. Meyer and J. Serra, "Contrast and Activity Lattice," Signal Processing, vol. 16, pp. 303-317, 1989.

[5]. J. D. Mendiola-Santibañez and I. R. Terol-Villalobos, "Morphological contrast mappings based on the flat zone notion," vol. 6, pp. 25-37, 2005.

[6]. A. Toet, "Multiscale contrast enhancement with applications to image fusion," Opt. Eng., vol. 31, no. 5, 1992.

[7]. S. Mukhopadhyay and B. Chanda, "A multiscale morphological approach to local contrast enhancement," Signal Process. vol.80, no.4, pp.685-696, 2000.

[8]. A. K. Jain, Fundamentals of Digital Images Processing. Englewood Cliffs, NJ: Prentice-Hall, 1989.

[9]. J. Short, J. Kittler, and K. Messer, "A comparison of photometric normalization algorithms for face verification," presented at the IEEE Int. Conf. Automatic Face and Gesture Recognition, 2004.

[10]. C. R. González and E.Woods, Digital Image Processing. Englewood Cliffs, NJ: Prentice Hall,1992.

[11]. R. H. Sherrier and G. A. Johnson, "Regionally adaptive histogram equalization of the chest,'IEEE Trans. Med. Imag., vol. MI-6, pp. 1-7, 1987.

[12]. A. Majumder and S. Irani, "Perception-based contrast enhancement of images," ACM Trans. Appl. Percpt., vol. 4, no. 3, 2007, Article 17. International Journal of Computer Science \& Information Technology (IJCSIT) Vol 4, No 1, Feb 201250

[13]. Z. Liu, C. Zhang, and Z. Zhang, "Learning-based perceptual image quality improvement for video conferencing," presented at the IEEE Int. Conf. Multimedia and Expo (ICME), Beijing, China, Jul. 2007.

[14]. J. Serra and P. Salembier, "Connected operators and pyramids," presented at the SPIE. Image Algebra and Mathematical Morphology, SanDiego, CA, Jul. 1993.

[15]. P. Salembier and J. Serra, "Flat zones filtering, connected operators and filters by reconstruction," IEEE Trans. Image Process., vol. 3, no.8, pp. 1153-1160, Aug. 1995.

[16]. P. Soille, Morphological Image Analysis: Principle and Applications. New York: Springer-Verlag, 2003.

[17]. H. Heijmans, Morphological Image Operators. New York: Academic, 1994

[18]. L. Vincent and E. R. Dougherty, "Morphological segmentation for textures and particles," in Digital Image Processing Methods, E. R. Dougherty, Ed. New York: Marcel Dekker, 1994, pp. 43-102. 